
zygotic embryos of Picea pungens

\author{
Jing Tao ${ }^{1 凶}$, Shigang Chen ${ }^{1}$, Caiyun $\mathrm{Qin}^{1}$, Qingmei $\mathrm{Li}^{1}$, Jufeng Cai ${ }^{1}$, Changbin Sun ${ }^{2}$, \\ Weiming Wang ${ }^{3}$ \& Yuhui Weng ${ }^{4}$
}

This study developed somatic embryogenesis protocols for Picea pungens (Engelm), an important ornamental species, including initiation, proliferation, maturation, germination, and acclimation. Somatic embryogenic tissues were induced from mature zygotic embryos of five families, with a frequency of $\geq 22 \%$ for each. Embryogenic tissues (ET) from 13 clones of three families were proliferated for one week, achieving an average rate of $179.1 \%$. The ET of 38 clones of three families were cultured in maturation medium for six weeks; 188 mature embryos on average were counted per gram ET cultured, of which $\geq \mathbf{8 1 . 1 \%}$ appeared normal, and each clone developed at least 28 normally matured embryos. A total of $69.9 \%$ or more of cotyledonary somatic embryos germinated normally and developed into normal emblings. The experiment of transplanting the emblings into a greenhouse had an average survival rate of $68.5 \%$. Considerable variation among and within families during initiation and proliferation was observed, but this variation decreased in the maturation and germination. Changing the concentration of plant growth regulator of the initiation medium did not significantly change the initiation frequency. We recommend incorporating these protocols into the current Picea pungens practical programs, although further research is essential to increase efficiencies and reduce cost.

Advantages of incorporating somatic embryogenesis (SE) into a forest genetic selection and deployment program have been well reported ${ }^{1-3}$. Successful examples in this regard can be found in many commercial species, particularly spruce, such as Picea abies ${ }^{4}$, Picea sitchensis ${ }^{5}$, Picea glauca $^{6-8}$, and Picea mariana ${ }^{6,9}$. The operational practice of SE techniques has been incorporated into the commercial forestry of Picea glauca and Picea mariana since $1990^{9,10}$.

Valued mainly for its appearance due to its unique, year-round silvery blue-green needle color, Picea pungens (Engelm.) has been planted extensively as an ornamental species in North America and Europe ${ }^{11}$. Picea pungens has been used in northeast China as an important ornamental species since $2000^{12,13}$. Demand for planting the species has been increasing in the past 20 years, and consequently, seed production areas have been established ${ }^{12}$. Planting Picea pungens using seeds, however, is associated with issues, including difficulty in retaining desirable parental tree characteristics, in particular needle color. Thus, propagation methods that retain ideal characteristics of selected genotypes and allow for efficient regeneration of plants on a large scale are ideal. Artificial vegetative propagation using cuttings or grafting produces the same genotype (clone) as the donor tree, and the effectiveness of these methods is often dependent on the age and physiological state of the donor plant, limiting its practical application in forestry. Given the success of SE in spruce ${ }^{9,10}$, vegetative propagation of Picea pungens via the SE method is becoming attractive since the method can overcome the problems associated with cuttings or grafting. An embryogenic cell line established from one seed can generate a high number of somatic embryos; therefore, SE can produce a large number of genetically identical plants within a short period. Nonetheless, SE is a complicated process of multiple steps, including initiation, proliferation, maturation, germination, and acclimation, and for a successful SE method, a satisfactory result is indispensable for each step.

Despite the great desire to apply SE techniques to produce Picea pungens seedlings, relevant studies have been limited in the literature. For this species, successful SE initiation and maturation were first reported by Afele et al. ${ }^{14}$, although their efficiencies were relatively low. In subsequent studies ${ }^{15,16}$, great improvement in efficiency was made in initiation and proliferation, but satisfactory results were not achieved in maturation or germination. Clearly, obstacles to SE embryo maturation and germination remain. Furthermore, to our knowledge, data for the subsequent step, acclimation, have been lacking, and such data are essential to assess the feasibility of

\footnotetext{
${ }^{1}$ Jilin Provincial Academy of Forestry Sciences, 3528 Linhe St., Changchun, Jilin, China. ${ }^{2}$ Changchun Academy of Forestry, 5840 Jingyue St., Changchun, Jilin, China. ${ }^{3}$ AbrorGen Inc., 2011 Broadbank Court, Ridgeville, SC 29472, USA. ${ }^{4}$ Arthur Temple College of Forestry and Agriculture, Stephen F. Austin State University, Nacogdoches, TX75965, USA.『email: taojing8116@126.com; wengy@sfasu.edu
} 


\begin{tabular}{|c|c|c|}
\hline Step & PGR concentration $(\mu \mathrm{M})$ & Supplement $(\mathrm{g} / \mathrm{L})$ \\
\hline Initiation & $\begin{array}{l}\text { Low: } \\
\text { BA } 1.25+2,4-D 2.5 \\
\text { BA } 2.5+2,4-D 5.0 \\
\text { Standard: } \\
\text { BA } 5.0+2,4-D 10.0 \\
\text { High: } \\
\text { BA 7.5 +2,4-D } 12.5 \\
\text { BA 7.5+2,4-D } 15.0 \\
\text { BA } 10.0+2,4-D 15.0 \\
\text { BA } 10.0+2,4-D 20.0\end{array}$ & $\begin{array}{l}\text { Sucrose } 10.0 \\
\text { Gelrite } 4.0 \\
\text { Glutamine } 0.5\end{array}$ \\
\hline \multirow[b]{2}{*}{ Proliferation } & $\begin{array}{l}\text { Liquid: } \\
\text { BA } 3.75+2,4-\mathrm{D} 6.25\end{array}$ & $\begin{array}{l}\text { Sucrose } 10.0 \\
\text { Glutamine } 0.5\end{array}$ \\
\hline & $\begin{array}{l}\text { Solid: } \\
\text { BA 7.5 + 2,4-D } 12.5\end{array}$ & $\begin{array}{l}\text { Sucrose } 10.0 \\
\text { Gelrite } 4.0 \\
\text { Glutamine } 0.5\end{array}$ \\
\hline Maturation & ABA 60.0 & $\begin{array}{l}\text { Sucrose } 60.0 \\
\text { Gelrite } 6.0 \\
\text { Glutamine } 0.5\end{array}$ \\
\hline Germination $^{\mathrm{a}}$ & & $\begin{array}{l}\text { Sucrose } 20.0 \\
\text { Gelrite } 6.0 \\
\text { Glutamine } 0.5\end{array}$ \\
\hline
\end{tabular}

Table 1. Plant growth regulator (PGR) treatments and concentrations and supplement contents were applied by somatic embryogenesis. Note that the base medium was half-strength Litvay's medium. ${ }^{\mathrm{a}}$ Contents for all half-strength Litvay's medium elements were reduced to half other than $\mathrm{KNO}_{3}, \mathrm{KH}_{2} \mathrm{PO}_{4}$, and Fe-EDTA.

using SE techniques in deployment. Overall, the techniques achieved thus far have not been sufficiently refined, particularly for maturation and the subsequent steps, limiting the application of SE to Picea pungens.

While modification and refinement of protocols continue to be an important aspect of SE research, other topics, such as genetic influence, can play an important role during the SE process. This has been confirmed in some spruce species s,9,17 $^{5}$ but is not well known for Picea pungens, since all previous studies used a single genotype ${ }^{13-15}$. The genetic effect on SE is complicated, depending on the SE stages ${ }^{16}$. An effective SE program for economically important tree species needs protocols that can be applied to a large number of genotypes.

A project to develop protocols for applying SE in Picea pungens breeding and deployment was initiated in late 2000 at the Jilin Academy of Forest Science and Research in NE China. Since SE protocols for Picea glauca and Picea mariana have been well refined and successfully incorporated into commercial seedling propagation in New Brunswick, Canada ${ }^{9}$, in the present study, we adopted those protocols as the foundation, with some adjustments, to Picea pungens. By using mature zygotes of Picea pungens as materials, all steps of the SE process, starting from embryo initiation to the transfer of emblings to the greenhouse, were evaluated. Thus, the objectives of the present study were (1) to develop protocols for the SE of Picea pungens and (2) to investigate whether SE efficiencies vary with family and clone. Initiation is key to spruce SE and may vary with changes in plant growth regulator (PGR) concentrations; thus, a third objective was to test the feasibility of improving initiation frequency by changing PGR concentrations.

\section{Materials and experiments}

In 2008, stored mature Picea pungens seeds from five families, representing diverse origins from New Brunswick (Family 113 and 8285450, gifted from New Brunswick Department of Natural Resources and National Tree Seed Center, Natural Resources Canada, respectively), Nova Scotia (200301, purchased from Nova Tree Seed Company, Inc., Truro, Nova Scotia), and Ontario (OSC, purchased from Ontario Seed Center, Waterloo, Ontario) in Canada and New York, USA (10910, purchased from TreeHelp.com, Buffalo, New York), were used as materials. Prior to the experiments, all seeds were stored at low temperature for two or more years.

Prior to embryo extraction, all seeds were submerged in $75 \%$ ethanol for $1 \mathrm{~min}$, rinsed three times in sterile water, soaked in $10 \%$ sodium hypochlorite with a small amount of Tween-20 for 30 min, rinsed five times in sterile water, and then kept in sterile water for $4 \mathrm{~h}$ to soften the seed coat. Mature zygotic embryos were extracted from seeds under a stereomicroscope. Experiments for key SE steps were then carried out, and details for the respective medium supplements and PGR concentrations are presented in Table 1 and for the experimental materials in Table 2.

Exp. 1: Initiation of embryogenic tissue. The initiation medium was half-strength Litvay's medium ${ }^{18}$ $(1 / 2 \mathrm{LM})$ supplemented with sucrose, L-glutamine, gelrite, and a (standard) PGR concentration combination containing 10.0 $\mu \mathrm{M}$ 2,4-dichlorophenoxyacetic acid (2,4-D) and 5.0 $\mu \mathrm{M}$ benzyl aminopurine (BA) (referred to as the standard PGR concentration; Table 1). To investigate the effects of PGR concentration, two PGR concentration treatments, low and high, were also tested. The low treatment included two concentration combinations containing $\leq 5.0 \mu \mathrm{M} 2,4-\mathrm{D}$ and $\leq 2.5 \mu \mathrm{M} \mathrm{BA}$, and the high treatment had four concentration combinations containing $\geq 12.5 \mu \mathrm{M} 2,4-\mathrm{D}$ and $\geq 7.5 \mu \mathrm{M}$ BA (Table 1 ). The $\mathrm{pH}$ of the medium was adjusted to 5.8 prior to sterilization.

All five families, with an average of 79 (range 75-94) mature zygotic embryos per family, were cultured on the initiation media in $100 \times 15-\mathrm{mm}$ Petri dishes containing approximately $25 \mathrm{ml}$ of medium (approximately 10-15 embryos per dish and approximately 10 dishes per family $\times$ PGR combination) in darkness at $24 \pm 1{ }^{\circ} \mathrm{C}$. 


\begin{tabular}{|l|l|l|l|l|l|}
\hline Step & Number of families & Number of clones per family & Total cultured $^{\mathbf{a}}$ & Total success $^{\mathbf{a}}$ & Rate $^{-}$ \\
\hline Initiation & 5 & 79 & 2902 & 979 & 0.34 \\
\hline Proliferation & 3 & 13 & 4.3 & 11.9 & 2.79 \\
\hline Maturation & 3 & 38 & 8324 & 6751 & 0.81 \\
\hline Germination & 2 & 13 & 633 & 443 & 0.70 \\
\hline Acclimation & 2 & 7 & 261 & 179 & 0.69 \\
\hline
\end{tabular}

Table 2. Summary of the number of families, the number of clones per family, the total cultured tissues, the total successful tissues and the success rate by somatic embryogenesis step. ${ }^{\text {a } U n i t s ~ f o r ~ t o t a l ~ c u l t u r e d ~ a n d ~}$ total success were the total number of zygotic embryos cultured and the number of embryos with established embryogenic tissues for initiation, weight ( $\mathrm{g}$ ) of embryogenic tissues before and after the liquid culture period for proliferation, the number of total matured and the number of normally matured embryos for maturation, the number of total matured embryos planted and the number of emblings developed for germination, the number of emblings planted, and the number of emblings that successfully developed for acclimation, respectively.

One month later, SE initiation was assessed by recording the success or failure of each cultured embryo. Success was defined for initiation of an embryo if embryogenic tissue (ET) was established. The initiation frequency (= number of embryos with successful initiation/number of cultured embryos) was then calculated to indicate initiation efficiency. Once an embryo had developed ET, it was given a clone identification number. Note that a clone (embryogenic line) in this study refers to embryogenic tissues initiated from a single embryo.

Exp. 2: Proliferation of embryogenic tissue. Once an ET clone was identified, a rapid proliferation of ET for cryopreservation and/or production was needed. This experiment used ET from 12 clones representing three families $(113,8285450$ and OCS) obtained in Exp. 1. ET was proliferated via a method of two steps: liquid suspension culture on liquid proliferation medium, followed by culture on solid proliferation medium. The base proliferation medium was the same as the initiation medium, but the PGR combination varied, being $3.75 \mu \mathrm{M}$ BA and 6.25 $\mu \mathrm{M}$ 2,4-D for liquid and 7.5 $\mu \mathrm{M}$ BA and $12.5 \mu \mathrm{M} 2,4-\mathrm{D}$ for solid cultures (Table 1).

Before transferring to the proliferation medium, ET was cultured on medium without BA and 2,4-D for seven days. Approximately 30-40 mg ET per clone was subcultured in flasks (10-15 mg ET per flask) with $50 \mathrm{ml}$ liquid proliferation medium for seven days. During this period, the flasks were shaken vigorously (121 RPM) to break up the tissue pieces into a fine suspension. The suspension in a flask was poured onto filter paper to remove liquid, and the filter paper with attached cells was placed onto the surface of fresh, solid proliferation medium. The tissues were cultured in darkness at approximately $24^{\circ} \pm 1{ }^{\circ} \mathrm{C}$ for two weeks and then subcultured every 14 days on the solid medium. ET weights for each flash were recorded before and after the liquid culture period, and the percentage increase $\left(=\frac{\text { after-before }}{\text { before }} \times 100\right)$ was used as the response variable for proliferation efficiency. Due to its difficult measurement, proliferation in solid culture was not recorded.

Exp. 3: Maturation of somatic embryos. This experiment was conducted using ET from 38 clones initiated from three families $(113,8285450$ and OCS) in Exp. 1 and 2. The maturation medium was 1/2 LM containing $6 \%$ sucrose, $0.6 \%$ gelrite, $0.05 \%$ glutamine, and $60 \mu \mathrm{M}$ abscisic acid (ABA) (Table 1 ).

Before transferring to the maturation medium, ET was subcultured on solid proliferation medium for two periods, each period being 7 days, and then cultured on proliferation medium without BA and 2,4-D for another seven days. ET clumps that grew vigorously were selected for maturation. 3-8 Petri dishes each with 6-8 clumps (approximately $0.5 \mathrm{~g}$ ) were prepared for each clone. Cultures were kept at approximately $24^{\circ} \mathrm{C}$, low light intensity $\left(5 \mathrm{~mol} / \mathrm{\mu m}^{2} / \mathrm{s}\right.$, cool white, fluorescent lamps GE F72T12/CW), and a 16-h photoperiod for six weeks without subculturing onto fresh medium. By the end of the culture period, the total number of normal embryos (1.5-2 mm in length, having 2-5 distinct cotyledons, embryonal root caps, and smooth hypocotyls) and abnormal embryos were counted and expanded on a per gram basis. The number of normally matured embryos per gram was used as the indicator of maturation efficiency.

Exp. 4: Germination and rooting. This experiment involved 13 clones representing two different families (8285450 and OCS) from Exp. 3. The germination medium was a modified $1 / 2$ LM paired with $2 \%$ sugar, $0.6 \%$ Gelrite, 0.05\% L-Glutamine and no PGRs (Table 1).

Matured embryos at the cotyledonary stage in Exp. 3 were isolated and placed onto germination medium (20 mature embryos per dish and approximately three dishes per clone) for culture under similar environmental conditions as for maturation. After 1 week of culture in darkness at $24 \pm 1{ }^{\circ} \mathrm{C}$, cotyledonary and small radicles appeared. After culture for the next four weeks in the light, normal emblings (plantlets germinated from somatic embryos with young hypocotyls of 1-2 cm and radicles of 2-3 cm) had developed. The total number of embryos planted and the number of emblings developed after the culture were recorded. The frequency $(=$ number of normal emblings/number of embryos planted) was calculated to indicate germination efficiency. 
Exp. 5: Acclimation. This experiment used six clones representing one family (8285450) and one clone from family OCS in Exp. 4. On average, 44 emblings per clone with properly developed radicles and hypocotyls were directly transferred into pots (filled with Jiffy peat pellets). For the first two weeks in the greenhouse, the emblings were kept under a 16 -h photoperiod at $25 \pm 5{ }^{\circ} \mathrm{C}$ and each embling plantlet was kept under a glass beaker to protect against dehydration. The emblings were grown until they reached a height of $10-15 \mathrm{~cm}(20-$ 25 weeks). The number of emblings that successfully developed was counted, and the survival rate (= number of successfully developed emblings/total number of planted emblings) was calculated as the indicator of acclimation efficiency.

Data analysis. In Exp 1, the response variable for initiation, the number of embryos with successful initiation out of the total number of embryos cultured (data in 'event/trial' type), was assumed to follow a binomial distribution and analyzed using the following models:

$$
y_{i j k}=\mu+F_{i}+T_{j}+F T_{i j}+P_{k(j)}+\varepsilon_{i j k},
$$

where $y_{i j k}$ was the event/trial in initiation of the kth petri dish of the ith family and jth PGR treatment and kth PGR concentration combination within the jth PGR treatment. $\mu$ was the overall mean, $F_{i}, T_{i}, P_{k(j)}$, and $F T_{i j}$ were the fixed effects of the family, PGA treatment, PGR combination within a treatment, and the interaction between $F$ and $T$, respectively, and $\varepsilon_{i j k l}$ was the random error.

The response variable of Exp 2, the percent ET increase of the kth dish of the jth clone within the ith family $\left(y_{i j k}\right)$ is continuous data and, was, therefore, analyzed using analysis of variance following the model below:

$$
y_{i j k}=\mu+F_{i}+C_{j(i)}+\varepsilon_{i j k},
$$

where $C_{j(i)}$ was the fixed effect of the jth clone within the ith family, and the others remained the same as explained previously.

In Exp. 3, the total number of normally matured embryos per gram ET cultured is count data and can be modeled using a Poisson distribution. The data were analyzed using Model [2] but paired with a negative binomial distribution, which is similar to the Poisson distribution but can handle the over-dispersion issue.

In Exp. 4 and 5, the response variable was the 'event/trial' type. The numbers of emblings over the embryos cultured on germination medium and the normal emblings developed over the total emblings transferred in acclimation were analyzed using Model [2], where all terms remained the same as previously described.

SAS programs ${ }^{19}$ were used in the analyses (i.e., logistic procedure for the 'event/trial' data, GLM procedure for the continuous data, and GENMOD procedure for the count data). Means and their standard errors (SE) were computed either on an original scale directly or transformed to the original scale via a link, depending on the data type. Multiple comparisons among levels of a factor were made using the Tukey method. Pearson correlations at the clonal level among efficiencies of proliferation, maturation, germination, and acclimation were tested for significance. Unless otherwise stated, the significance level in this paper refers to $\alpha=0.05$.

Research involving plants. The authors declare that this study on plants in this study, including the collection of plant material, complies with relevant institutional, national, and international guidelines and legislation.

\section{Results}

Two weeks post-inoculation, light brown tissues appeared on the zygotic embryo explants, and after another 1-2 weeks, embryos started to produce translucent early embryogenic tissue (Fig. 1a). While all families responded to the initiation media, the frequency differed among families (Table 3; Fig. 2a). The frequencies were comparable (ranging from 0.23 to 0.27 ) for all families other than family 10910, which had a significantly higher frequency of 0.68 , resulting in an overall initiation frequency of 0.34 (Table 2). The impact of PGR treatment on ET induction (Table 3) was insignificant, as was that among the combinations within a treatment (Table 1). Nevertheless, the highest frequency was observed under the high PGR treatment (0.34), which was closely followed by the standard (0.32) and low (0.31) treatments. Family responded similarly to PGR treatments, as demonstrated by their insignificant interaction (Table 3 ).

The initiated ET continued proliferating in the proliferation medium (Fig. 1b). By the end of the liquid culture period, all clones increased their ET fresh weight (Fig. 1c), on average by $179.1 \%$ (Table 2). The rate differed significantly by family (Table 4), ranging from 138.3 to $266.0 \%$ (Fig. 2b). The rate also varied greatly within families (Table 4); for example, among the eight clones representing the family 8285450 , the proliferation rate ranged from 8.7 to $192.5 \%$ (Table 5).

Within one month after transfer, the ET started to develop mature somatic embryos, and after two months, the mature embryos were fully developed (Fig. 1d). Many mature embryos were identified, with an average of 188 embryos per gram ET, and the majority $(81.1 \%)$ of the embryos appeared normal (Table 2 ). While normally matured embryos were observed in all families and clones (Fig. 2c; Table 5), the differences in number were barely significant between families, but were significant within families (Table 4). For family 8285450, the average number of normally matured embryos by clone varied from 28 to 977 (Table 5). Even at the family level, their practical differences were large; the family OCS developed 230 mature embryos per gram ET, which was more than doubled that of the family 113 (Fig. 2c).

On the germination medium, the somatic embryos elongated their radicle and hypocotyl quickly, followed by the growth of a shoot and a root (Fig. 1e). All families and clones responded to the process; after four weeks of culture, the average frequency of developing emblings was 0.70 (Table 2). Even though the frequencies were 

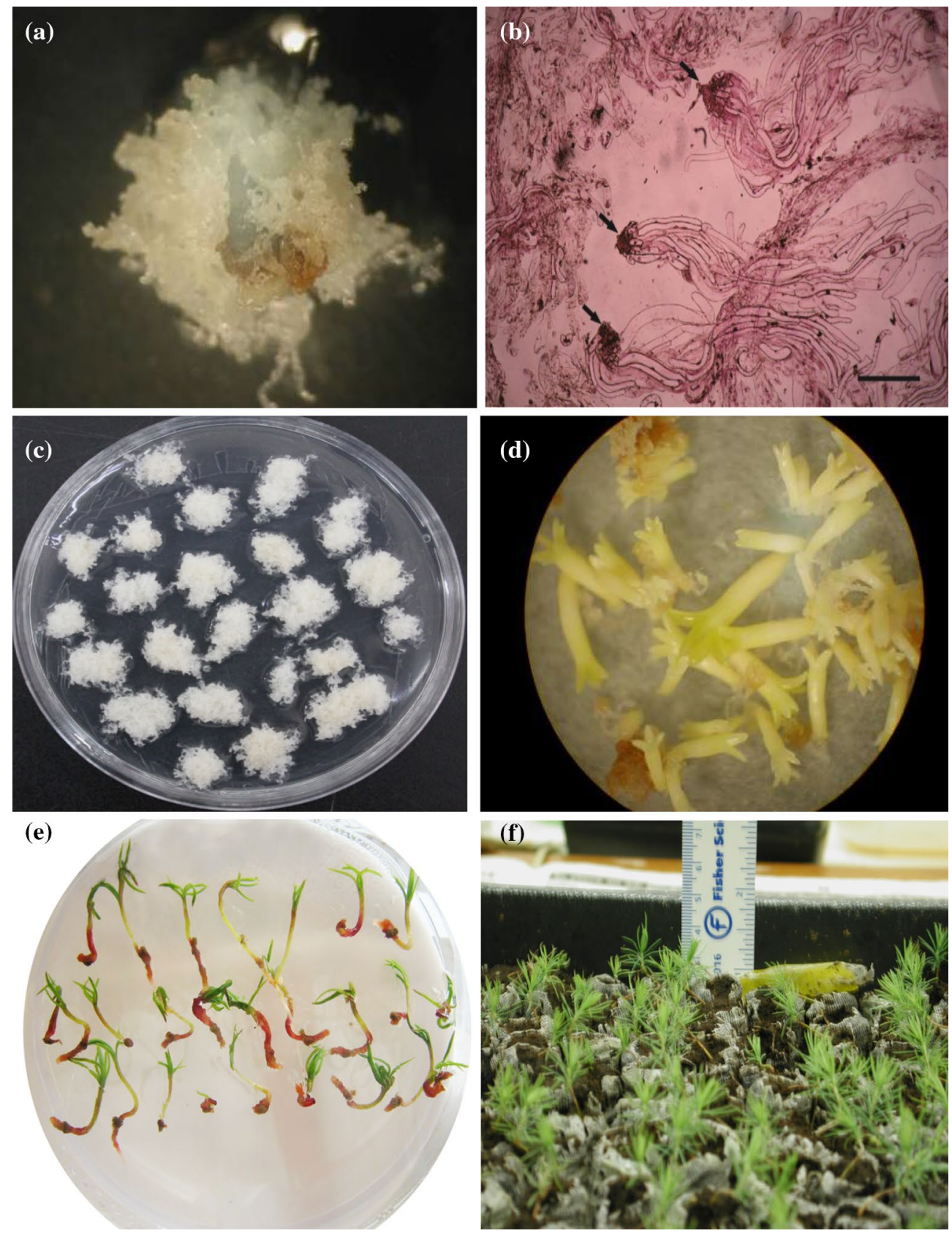

Figure 1. Somatic embryogenesis in Picea pungens: (a) initiation of embryogenic cell mass; (b) embryogenic tissues under a microscope after 14 days proliferation culture (bar $=50 \mu \mathrm{m})$; (c) embryogenic tissues on solid proliferation medium; (d) normally matured somatic embryos under mature conditions; (e) embryo germination and rooting; and (f) growing embryo seedlings for family 8285450.

\begin{tabular}{|l|l|l|l|}
\hline Source & DF & Chi-Square & Pr $>$ ChiSq \\
\hline Family & 4 & 287.48 & $<0.0001$ \\
\hline Treatment & 2 & 3.23 & 0.1989 \\
\hline Combination (treatment) & 4 & 0.28 & 0.9910 \\
\hline Family $\times$ treatment & 8 & 8.62 & 0.3750 \\
\hline
\end{tabular}

Table 3. Results of analysis of variance on embryo initiation (Exp. 1). 



Figure 2. Average and standard error by family: (a) initiation frequency; (b) proliferation rate (\% increase); (c) the number of mature embryos per gram embryo tissue; (d) germination frequency; and (e) embling survival rate in acclimation.

\begin{tabular}{|c|c|c|c|c|c|c|c|c|c|c|c|c|}
\hline \multirow[b]{2}{*}{ Source of variation } & \multicolumn{3}{|c|}{ Proliferation } & \multicolumn{3}{|c|}{ Maturation } & \multicolumn{3}{|c|}{ Germination } & \multicolumn{3}{|c|}{ Acclimation } \\
\hline & $d f^{\mathrm{a}}$ & F value & Pr & df & Chi-Square & $\operatorname{Pr}$ & df & Chi-Square & Pr & df & Chi-Square & Pr \\
\hline Family & 2 & 4.34 & 0.027 & 2 & 5.80 & 0.055 & 1 & 0.09 & 0.769 & 1 & 10.36 & 0.001 \\
\hline Clone (family) & 9 & 2.66 & 0.033 & 24 & 45.59 & 0.005 & 9 & 6.89 & 0.649 & 4 & 14.96 & 0.005 \\
\hline
\end{tabular}

Table 4. Results of analysis of variance on embryo tissue proliferation, maturation, germination and

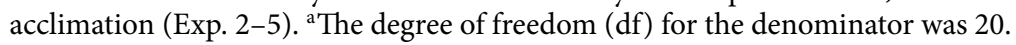




\begin{tabular}{|l|l|l|l|}
\hline \multirow{2}{*}{ Phase } & \multicolumn{3}{|l|}{ Family } \\
\cline { 2 - 4 } & $\mathbf{8 2 8 5 4 5 0}$ & OCS & $\mathbf{1 1 3}$ \\
\hline Proliferation (\%) & $8.68-192.50$ & $214.29-308.33$ & $176.00-366.67$ \\
\hline Maturation (number) & $27.78-976.82$ & $144.00-699.97$ & $50.94-475.00$ \\
\hline Germination & $0.37-0.83$ & $0.27-0.83$ & $0.59-0.79$ \\
\hline Acclimation (\%) & $56.67-87.50$ & & $48.94^{\mathrm{a}}$ \\
\hline
\end{tabular}

Table 5. Clonal range of embryo tissue proliferation, maturation, germination and acclimation, sorted by family. ${ }^{a}$ Only one clone was tested.

\begin{tabular}{|l|l|l|l|}
\hline Step & Maturation & Germination & Acclimation \\
\hline Proliferation & -0.06 & -0.44 & -0.55 \\
\hline Maturation & & -0.10 & -0.11 \\
\hline Germination & & & -0.04 \\
\hline
\end{tabular}

Table 6. Pearson correlation coefficients among clonal averages in proliferation, maturation, germination, and acclimation. Note that none of the coefficients significantly differed from zero at $\alpha=0.05$.

statistically comparable between and within families (Table 4), the actual differences were substantial [i.e., for family 8285450 , the clonal frequency varied from 0.37 to 0.83 (Table 5)].

In Exp 5, for acclimation, the emblings developed in the germination culture were directly transferred to pots (filled with Jiffy peat pellets) in a greenhouse environment (Fig. 1f). On average, 68.5\% of the transferred emblings survived (Table 2). The survival rate varied with family and clones within a family (Table 3). Family 8285450 (74.5\%) had a significantly higher survival than OCS (50.4\%) (Fig. 2e). At the clonal level, the average survival rate of the family 8285450 ranged from 56.7 to $87.5 \%$.

No particular patterns stood out in efficiency among proliferation, maturation, germination, or acclimation at the family level (Fig. 2). At the clone level, no calculated correlation coefficient values significantly differed from zero. However, proliferation correlated negatively with germination $(\mathrm{r}=-0.44)$ and acclimation $(\mathrm{r}=-0.55)$ to some extent (Table 6).

\section{Discussion}

In this study, efforts were made to develop practical SE protocols for Picea pungens. We used the protocols for Picea glauca and Picea mariana ${ }^{8,9}$ as the base and made some modifications based on preliminary trials (data not shown) in order to improve efficiency. Important modifications included: (1) a two-stage proliferation process, first in a liquid medium and then on a solid medium, which is different from that of Adams et al. ${ }^{9}$, in which a one-stage, solid medium proliferation was used. Liquid suspension cultures of conifers were preferred for proliferation since they can multiply at higher rates than solid medium culture ${ }^{20}$. In this study the liquid proliferation culture for seven days increased ET by $179 \%$ (Fig. 2); and (2) the ABA concentration of maturation medium was increased to $60 \mu \mathrm{M}$ (Table 1), whereas that of Adams et al. ${ }^{9}$ used $8 \mu \mathrm{M}$, a sevenfold increase. A wide range (0.1-25 mg/L) of ABA has been used for the development and maturation of conifer somatic embryos ${ }^{21}$, but the suitable level varied with species and genotype. For Picea pungens, the optimal ABA requirement for maturation varied; Afele et al. ${ }^{14}$ found that it depended on PGR treatment in the initiation medium (i.e., 7.6 $\mu \mathrm{M} \mathrm{ABA}$ if the initiation medium containing $10 \mu \mathrm{M}$ NAA or 2,4-D and $5 \mu \mathrm{M}$ BA) but Sun and Jia ${ }^{16}$ recommended a much higher concentration $(2 \mathrm{mg} / \mathrm{L})$. Overall, results following all the steps in this study are promising having achieved efficiencies to levels similar to those for Picea glauca $^{17}$ and Picea mariana $a^{9,10,17}$, one of the most successful spruce SE commercial programs. In supporting our results, the protocols for Picea glauca ${ }^{8}$ have also been successfully applied to SE of Picea sitchensis ${ }^{5}$.

At the species level, this study represents great improvements in SE efficiencies and techniques relative to previous SE studies on Picea pungens. For this species, the first paper ${ }^{14}$ reported an initiation frequency of 0.13 , which was then improved upon to $0.21-0.45$ in subsequent studies ${ }^{15,16}$. Similarly, great progress has been made in proliferation, with the proliferation rate improving from approximately $15^{15}$ to $130 \%$. However, in the subsequent steps of maturation and germination, the majority of the embryos appeared abnormal and failed to develop roots ${ }^{16}$. These studies compared efficiencies among various basal media or PGR concentrations and their results varied. For example, DCR medium was the best for the initiation in one study ${ }^{16}$, while others supported the use of $1 / 2$ LM medium ${ }^{14,15}$. Compared with previous studies ${ }^{14-16}$, the present study was not found to be inferior in terms of initiation or proliferation, and also showed substantial improvements in maturation and germination. Additionally, the present study directly transplanted the emblings from the Petri dishes into containers in a greenhouse environment and is the first demonstration of successful acclimation with satisfactory survival rates. The survival rates, $74 \%$ for family 8285450 (Fig. 2) and $87.5 \%$ for its best clone (Table 5), were comparable to those reported for Picea sitchensis $(67 \%)^{5}$, Picea abies $(77 \%)^{22}$, Picea glauca and Picea mariana (above $\left.80 \%\right)^{10}$. Since physicochemical cultural conditions were not always the same among the present and previous studies, it 
is difficult to attribute the superiority of this study to any particular factors. One thing, which is crucial but often ignored in developing protocols for a SE system, is that physicochemical conditions of one step may affect results of its subsequent steps. While high auxin/cytokinin combinations facilitated SE initiation ${ }^{16}$, there might have a negative effect on the subsequent cultures, in particular on the ability of the somatic embryos to differentiate into plantlets ${ }^{14}$. Also, high ABA concentrations during maturation can inhibit germination. This might partly contribute to the failure in germination in Ref. ${ }^{16}$, where a high ABA amount $(2.0 \mathrm{mg} / \mathrm{L})$ was used in maturation. The current study evaluated the SE system involving all key steps and the promising results for each step suggest the practical significance of the protocols, although there may still be room for improvement.

Plant growth regulator is essential for SE initiation. A few studies have investigated the effects of various PGR concentrations on initiation of Picea pungens, but their results varied, with some ${ }^{14,15}$ showing insignificant effects and others ${ }^{16}$ a reversal conclusion. The current study supports ${ }^{14,15}$, with no significant difference being found among and within PGR treatments (Table 3), although a trend of slightly increasing initiation frequency with increasing PGR concentration was also found. Recommended PGR concentration combinations for Picea pungens initiation varied: One recommended a PGR concentration combination of $5.0 \mathrm{mg} / \mathrm{L} 2,4-\mathrm{D}, 4.0 \mathrm{mg} / \mathrm{L}$ $\mathrm{BA}$, and $2.0 \mathrm{mg} / \mathrm{L} \mathrm{KT}^{16}$, a concentration combination similar to our high treatment PGR combination (Table 1), whereas others ${ }^{14,15}$ suggested a PGR concentration combination of $9 \mu \mathrm{M} 2,4$-D or NAA and $4.5 \mu \mathrm{M}$ BA, which is similar to our standard treatment. It appears that a wide range of PGR concentration combinations could be effective for Picea pungens, although higher concentrations might be preferred for initiation based on our results. However, high PGR concentrations might increase browning and aging of cultured tissues ${ }^{15}$. The impact of PGR on initiation may be species specific; for example, in Pinus strobus, the initiation frequency increases from an average of $0.20-0.53$ by reducing the $2,4-\mathrm{D}$ and BA concentrations from 9.5 to $2.2 \mu \mathrm{M}$ and from 4.5 to $2.2 \mu \mathrm{M}$, respectively ${ }^{23}$.

The capacity of SE is often genetically determined, and a standard protocol for each SE step may not be equally effective for all genotypes ${ }^{9,17,24-26}$. While all tested families and clones were responsive in each step in the current study, the variation among and within families was substantial, suggesting that, while the wide application of the protocols is plausible, low efficiencies may occur for some genotypes. Note that SE differences among genotypes in the current study might be underestimated due to the limited numbers of families and clones being tested. Clearly, the effect of genotype on the success of SE in the species should be emphasized, and more studies involving more genotypes are required before a definitive conclusion can be derived. The variation varied by SE step: It was significant in initiation and proliferation and became less significant or insignificant in the subsequent steps of maturation and germination (Table 4). Thus, to improve SE efficiency, genetic selection at the initiation or proliferation steps would be more effective. This pattern is in line with the discovery in Picea glauca that genetic control was strong in the initiation phase, but its influence steadily declined through the proliferation, maturation, and germination phases ${ }^{17}$. Even for maturation or germination, where variation among and within families was statistically insignificant (Table 4), the biological significance of the variation might be greater than was statistically evident. While a protocol applicable to a broad range of genotypes is indispensable for commercial timber species, a practical program with species of high ornamental value often works with a limited number of genotypes of great commercial value. The significant effect of genetic background reveals that future focus should be on developing more specific media for important seed families or clones of Picea pungens.

A satisfactory efficiency for each step is essential for a practical SE program and, thus, negative relationships in efficiency between steps among genotypes may compromise the overall SE efficiency. Selecting clones of higher proliferation rates might be accompanied by a decrease, although statistically insignificant, in efficiencies of germination and acclimation (Table 6). Overall, the observed correlation among the SE steps was weak in general at both the family (Fig. 2) and clonal levels (Table 6), suggesting that genetic selection at one step will not negatively affect the efficiencies of other steps substantially. In Picea glauca, no correlation was found between the frequencies of SE initiation and maturation, or germination rates ${ }^{27}$, which parallels our finding in this study. Note that, however, the observed correlations in the present study might be spurious owing to small sample sizes. In terms of genetic selection efficiency, this is an important topic deserving further investigation by including more genotypes.

Currently, Picea pungens is mainly propagated by seeds either imported from North America or collected from seed production areas. For this species, seed production is unpredictable, varying from year to year, and, importantly, there is no guarantee that the favorable silvery blue color of needles in parents can be retained in their offspring via seed propagation, a drawback for ornamental species. Given the satisfactory results in the current study and the advantages of SE in deployment, incorporating the SE protocols described here into a genetic selection and propagation system for Picea pungens is promising. ET can be induced from mature embryos of seeds, with some being used for SE plant production for testing and others for cryopreservation; a tested superior genotype can immediately be recultured and mass propagated via SE. We used mature zygotic embryos as explants, which may reduce the efficiency of spruce SE in general ${ }^{5,17,27}$, is more useful operationally, as they can be extracted from seeds stored in seed banks or collected from seed production areas.

The present work has successfully established SE protocols for Picea pungens. Even though the SE protocols were appropriate overall, in order to incorporate the techniques into a commercial forestry program of Picea pungens, more studies are needed. The protocols described here might be suboptimal and can be improved in efficiency. In addition to further modifying the SE protocols, future research should concentrate on the cryopreservation of ET as well as subsequent topics, such as somaclonal variation, somatic embryo recovery, and seedling growth in the field. Another issue is high cost, which may be reduced by automating SE steps ${ }^{28}$. The high cost of SE plants can be a limitation for any commercial species, but for ornamental species, such as Picea 
pungens, a high price for high-quality trees can always be commanded. Overall, the results of this study are encouraging in terms of efficiency, and the protocols can be used as a foundation for further commercializing SE techniques of Picea pungens.

Received: 29 May 2021; Accepted: 8 September 2021

Published online: 24 September 2021

\section{References}

1. Högberg, K. A., Ekberg, I., Norell, L. \& von Arnold, S. Integration of somatic embryogenesis in a tree breeding programme: A case study with Picea abies. Can. J. For. Res. 28, 1536-2545 (1998).

2. Park, Y. S. Implementation of conifer somatic embryogenesis in clonal forestry: Technical requirements and deployment considerations. Ann. For. Sci. 59, 651-656 (2002).

3. Lelu-Walter, M. A. et al. Somatic embryogenesis in forestry with a focus on Europe: State-of-the-art, benefits, challenges and future direction. Tree Genet. Genomes 9, 883-899 (2013).

4. Egertsdotter, U. \& von Arnold, S. Development of somatic embryos in Norway spruce. J. Exp. Bot. 49, 155-162 (1998).

5. Fenning, T., O’Donnell, M. \& Connolly, T. An assessment of somatic embryogenesis and cryo-preservation methods with a wide range of Sitka spruce breeding material from the UK. Plant Cell Tissue Organ Cult. 131, 483-497 (2017).

6. Attree, S. M., Tautorus, T. E., Dunstan, D. I. \& Fowke, L. C. Somatic embryo maturation, germination, and soil establishment of plants of black and white spruce (Picea mariana and Picea glauca). Can. J. Bot. 68, 2583-2589 (1990).

7. Tremblay, F. M. Somatic embryogenesis and plantlet regeneration from embryos isolated from stored seeds of Picea glauca. Can. J. Bot. 68, 236-242 (1990).

8. Park, Y. S., Barrett, J. D. \& Bonga, J. M. Application of somatic embryogenesis in high-value clonal forestry: Deployment, genetic control, and stability of cryopreserved clones. In Vitro Cell Dev. Biol. Plant 34, 231-239 (1998).

9. Adams, G. W., Doiron, M. G., Park, Y. S., Bonga, J. M. \& Charest, P. J. Commercialization potential of somatic embryogenesis in black spruce tree improvement. For. Chron. 70, 593-598 (1994).

10. Adams, G. W., Kunze, H. A., McCartney, A., Millican, S. \& Park, Y. S. An industrial perspective on the use of advanced reforestation stock technologies. In Vegetative Propagation of Forest Trees (eds Park, Y. S. et al.) 323-334 (Seoul, National Institute of Forest Science, 2016)

11. Fechner, G. H. Silvical Characteristics of Blue Spruce. (U.S. Dept. of Agriculture, Forest Service, Rocky Mountain Forest and Range Experiment Station, 1984).

12. Tao, J. et al. Introduction of cold-tolerant color trees in Northern China. J. Chin. Urban For. 5, 16-18 (2007) (In Chinese with English abstract).

13. Wang, X. C., Tao, J., Chen, S. G., Li, Q. M. \& Zhang, K. M. Introduction study on color tree species with cold endurance in Northeast China. Jilin For. Sci. Technol. 36, 6-8 (2007) (In Chinese with English abstract).

14. Afele, J. C., Senaratna, T., McKersie, B. D. \& Saxena, P. K. Somatic embryogenesis and plant regeneration from zygotic embryo culture in blue spruce (Picea pungens Engelman). Plant Cell Rep. 11, 299-303 (1992).

15. Hazubska-Przybyl, T. \& Bojarczuk, K. Somatic embryogenesis of selected spruce species (Picea abies, P. omorika, P. pungens 'Glauca' and P. breweriana). Acta Soc. Bot. Pol. 77, 189-199 (2008).

16. Sun, J. S. \& Jia, G. X. Somatic embryogenesis of Picea pungens Engelmann. J. Beijing For. Univ. 32, 44-51 (2010) (In Chinese with English abstract).

17. Park, Y. S., Pond, S. E. \& Bonga, J. M. Initiation of somatic embryogenesis in white spruce (Picea glauca): Genetic control, culture treatment effects, and implications for tree breeding. Theor. Appl. Genet. 86, 427-436 (1993).

18. Litvay, J. D., Verma, D. C. \& Johnson, M. A. Influence of a loblolly pine (Pinus taeda L.) culture medium and its components on growth and somatic embryogenesis of the wild carrot (Daucus carota L.). Plant Cell Rep. 4, 325-328 (1985).

19. SAS Institute Inc. SAS/STAT 13.2 User's Guide. (SAS Institute Inc., 2014).

20. von Arnold, S., Sabala, I., Bozhkov, P., Dyachok, J. \& Filonova, L. Developmental pathways of somatic embryogenesis. Plant Cell Tissue Organ Cult. 69, 233-249 (2002).

21. Gupta, P. K. \& Grob, J. Somatic embryogenesis in conifers. In Somatic Embryogenesis in Woody Plants Vol. 1 (eds Jain, S. M. et al.) 81-98 (Kluwer Academic Publishers, 1995).

22. Tikkinen, M., Varis, S. \& Aronen, T. Development of somatic embryo maturation and growing techniques of Norway spruce emblings towards large-scale field testing. Forests 9, 325. https://doi.org/10.3390/f9060325 (2018).

23. Klimaszewska, K., Park, Y. S., Overton, C., MacEacheron, I. \& Bonga, J. M. Optimized somatic embryogenesis in Pinus strobus L. In Vitro Cell Dev. Biol. Plant 37, 392-399 (2001).

24. Niskanen, A. M. et al. Effect of parent genotype on somatic embryogenesis in Scots pine (Pinus sylvestris). Tree Physiol. 24, 1259-1265 (2004)

25. MacKay, J. J., Becwar, M. R., Park, Y. S., Corderro, J. P. \& Pullman, G. S. Genetic control of somatic embryogenesis initiation in loblolly pine and implications for breeding. Tree Genet. Genomes 2, 1-9 (2006).

26. Cheliak, W. M. \& Klimaszewska, K. Genetic variation in somatic embryogenic response in open-pollinated families of black spruce. Theor. Appl. Genet. 82, 185-190 (1991).

27. Park, Y. S., Pond, S. E. \& Bonga, J. M. Somatic embryogenesis in white spruce (Picea glauca): Genetic control in somatic embryos exposed to storage, maturation treatments, germination and cryopreservation. Theor. Appl. Genet. 89, 742-750 (1994).

28. Egertsdotter, U., Ahmad, I. \& Clapham, D. Automation and scale up of somatic embryogenesis for commercial plant production, with emphasis on conifers. Front. Plant Sci. https://doi.org/10.3389/fpls.2019.00109 (2019).

\section{Acknowledgements}

Part of this work was carried out at the SE lab of Canada Forest Service in Fredericton, New Brunswick, Canada, and Dr. Yill Sung Park and Mr. Ian Maceacheron provided great help/valuable suggestions for the work. We greatly appreciated their help/suggestions. The work was supported by the China Forestry Science and Technology Demonstration Project "Extension of High-efficient Propagation Techniques of Spruce" (JLT202110), Overseas Training Program of the State Administration of Foreign Experts Affairs "Techniques of Conifer Somatic Embryogenesis" (CG2007220005), and State Forestry Administration 948 Program of Introduction of International Advanced Agricultural Science and Technology project "Introduction of the Industrialization Technology for Spruce Somatic Embryogenesis" (2006-4-51). Partial support for this work was received from the National Institute of Food and Agriculture, USDA, McIntire-Stennis project. 


\section{Author contributions}

J.T. proposed the concept and designed the study. S.C., C.Q., Q.L., J.C., and C.S. implemented the study and collected the data. Y.W. and J.T. analyzed the data. J.T., Y.W., and W.W. drafted the manuscript. Y.W. and W.W. revised the manuscript. J.T. and Y.W. provided fund support.

\section{Competing interests}

The authors declare no competing interests.

\section{Additional information}

Correspondence and requests for materials should be addressed to J.T. or Y.W.

Reprints and permissions information is available at www.nature.com/reprints.

Publisher's note Springer Nature remains neutral with regard to jurisdictional claims in published maps and institutional affiliations.

(c) (i) Open Access This article is licensed under a Creative Commons Attribution 4.0 International License, which permits use, sharing, adaptation, distribution and reproduction in any medium or format, as long as you give appropriate credit to the original author(s) and the source, provide a link to the Creative Commons licence, and indicate if changes were made. The images or other third party material in this article are included in the article's Creative Commons licence, unless indicated otherwise in a credit line to the material. If material is not included in the article's Creative Commons licence and your intended use is not permitted by statutory regulation or exceeds the permitted use, you will need to obtain permission directly from the copyright holder. To view a copy of this licence, visit http://creativecommons.org/licenses/by/4.0/.

This is a U.S. Government work and not under copyright protection in the US; foreign copyright protection may apply 2021 